\section{Be aware: new rules for corticosteroids!}

\author{
Lars Engebretsen (1) 1,2
}

A paper in this issue on 'A novel approach to improve detection of glucocorticoid doping in sport with new guidance for physicians prescribing for athletes' by Ventura et $a l^{1}$ is important for every practising physician and physical therapist who is treating high level athletes. As of 2022 (remember; prior to the Games in Beijing), new rules will be in effect, and you need to be aware of them. This review evaluates the current glucocorticoid (GC) anti-doping regulations as defined by the WADA and presents a novel approach for defining permitted and prohibited use of GCs in sport based on the potential for performance enhancement and risk to health. Revised and substance-specific laboratory reporting values are presented in this paper to better distinguish between prohibited and permitted use in sport. ${ }^{2}$ In addition, washout periods are presented to enable clinicians to avoid the risk of athletes testing positive on a doping test.

The systemic effect of GCs following injectable and other routes of administration presents a potential both to improve performance and to cause harm to health in athletes. This paper presents a novel approach to a 'contentious' subject in antidoping-GCs injections. GCs have been 'contentious' in sport for years. When the systemic use of GCs was prohibited in sport by the IOC in 1986, the importance of GCs in clinical medicine was the primary reason why the development of Therapeutic Use Exemptions (TUEs) became necessary.

The paper has two components. Primarily, it is an attempt to categorise injected and other routes of GC in daily cortisol-equivalent doses and propose doses and routes of administration of GC that the authors suggest are potentially performance enhancing. Subsequently, the authors detail the extensive

'Department of Ortho surg, Oslo University Hospital, Oslo, Norway

${ }^{2}$ Department of Sports Medicine, Norwegian School of Sports Sciences, Oslo Sports Trauma Research Center, Oslo, Norway

Correspondence to Dr Lars Engebretsen, Oslo Sports Trauma Research Center, Department of Sports Medicine, Norwegian School of Sport Sciences, PB 4014 Ullevaal Stadion, N-0806 Oslo, Norway;

lars.engebretsen@medisin.uio.no research that has been undertaken to recommend modified laboratory reporting levels (RLs) for different GCs and suitable washout periods to enable clinicians to use GCs safely and to avoid the risk of athletes testing positive for a doping test. It is important to note that periarticular and peritendinous as well as intra-articular injections are classified as any other injection (since the serum level of GCs changes after an intraarticular injection). None of the inhaled, intranasal, dermal or other topical GCs when used at their maximum licensed approved doses are expected to exceed the $32 \mathrm{mg} /$ day threshold.

To improve the specificity of the RLs, WADA has sponsored excretion studies indicating that when GCs were administered by local injections (ie, intraarticular), the urinary concentrations were like those of other prohibited routes, indicating similar absorption and systemic exposure of the drug. Additionally, a single RL was not suitable for all GCs. The consequence of this is that higher vigilance is needed by the sport physicians and physical therapists using these drugs.

The use of systemic GCs by athletes is one of the the most common reasons why TUEs are sought by elite athletes. Reasons for GCs use include asthma and exercise-induced bronchoconstriction, ${ }^{3}$ autoimmune conditions, acute traumatic swelling and oedema, inflammatory bowel disease and other uncommon conditions such as Addison's disease, congenital adrenal hyperplasia and others. GCs are widely used in sports medicine, mainly to treat musculoskeletal injuries and asthma. GCs administered by local injection can be an important and effective option in the treatment plan of some musculoskeletal conditions commonly encountered in sports medicine.

The naturally occurring GC hormones and their synthetic analogues possess a wide range of potencies and pharmacokinetic properties. The body produces a daily output of endogenous GC (cortisol), typically $18-22 \mathrm{mg} /$ day and hence this amount of GC should not be regarded as performance enhancing, although abnormally low cortisol levels would likely impair performance. Administering GC drugs can result in a total GC exposure (exogenous+endogenous) that exceeds the upper threshold for normal daily physiological GC production and, hence, can potentially be performance enhancing whereas GC drug use that does not exceed this upper physiological threshold can reasonably be regarded as not performance enhancing.

Acceptable use is defined as a dose of exogenous GC (cortisol equivalent dose $\leq 5.28 \mathrm{mg} /$ day) that when added to normal physiological daily GC exposure $(26.4 \mathrm{mg} /$ day $)$ results in a total exposure (endogenous+exogenous) without reaching supraphysiological levels (cortisol equivalent dose $>32 \mathrm{mg}$ / day).

Unacceptable use is defined as a dose of exogenous GC that is equivalent to a dose demonstrated to be performance enhancing (eg, dexamethasone $4 \mathrm{mg}$, equivalent to $32.6 \mathrm{mg}$ cortisol) (see figure 1 in the paper). This is also $\approx 6$ times the $5.28 \mathrm{mg} /$ day acceptable dose defined above and results in a total GC exposure (26.4 mg endogenous + $32.6 \mathrm{mg}$ exogenous) of $\approx 60 \mathrm{mg} / \mathrm{day}$. Oral and injectable routes (eg, intravenous, intramuscular, subcutaneous, intra-articular) when used at their approved doses are likely to produce total GC exposures of $>32 \mathrm{mg} /$ day except at the lowest doses that are seldom used clinically. Up to now, a general urinary RL of $30 \mathrm{ng} / \mathrm{mL}$ was used to differentiate between permitted and prohibited administrations of all GCs. However, different studies have shown the need to establish compoundspecific RLs given the diversity of administration routes and doses, as well as pharmacokinetic and pharmacodynamic properties between the different GCs.

Washout periods for oral and injectable routes were defined considering the time of detection using the RLs established for each GC (see table 3). The washout period refers to the time taken from the last dose to the time of the in-competition period (midnight beginning the first day of the in-competition period) in order to reduce the GC concentration in the urine below the RLs. The maximum detection times obtained in each study are indicated in table 2. This is important, please read and place it on your office wall, stick it to your medical bag, and so on. The main issue; BE AWARE of the changes from 1 January 2022!

Twitter Lars Engebretsen @larsengebretsen 


\section{Editorial}

Contributors The corresponding author is fully and alone responsible for this editorial. LE developed the editorial idea and is responsible for the writing and final approval of the manuscript.

Funding The authors have not declared a specific grant for this research from any funding agency in the public, commercial or not-for-profit sectors.

Competing interests $L E$ is the editor for BJSM IPHP and co-chair of the IOC Medical and Scientific Commission.

Patient consent for publication Not required.

Provenance and peer review Not commissioned; externally peer reviewed. (c) Author(s) (or their employer(s)) 2021. No commercial re-use. See rights and permissions. Published by BMJ.

\section{(A) Check for updates}

To cite Engebretsen L. Br J Sports Med 2021;55:575-576.

Accepted 8 April 2021

Published Online First 27 April 2021

Br J Sports Med 2021;55:575-576. doi:10.1136/bjsports-2021-104099

ORCID iD

Lars Engebretsen http://orcid.org/0000-0003-2294921X

\section{REFERENCES}

1 Ventura R, Daley-Yates P, Mazzoni I. A novel approach to improve detection of glucocorticoid doping in sport with new guidance for physicians prescribing for athletes. British J Sports Med 2021. doi:10.1136/ bjsports-2020-103512

2 Vernec A, Slack A, Harcourt PR, et al. Glucocorticoids in elite sport: current status, controversies and innovative management strategies-a narrative review. Br I Sports Med 2020;54:8-12.

3 Allen H, Backhouse SH, Hull JH, et al. Anti-Doping policy, therapeutic use exemption and medication use in athletes with asthma: a narrative review and critical appraisal of current regulations. Sports Med 2019;49:659-68 